\title{
Corrigendum: Reduced IFN $\lambda 4$ activity is associated with improved HCV clearance and reduced expression of interferon-stimulated genes
}

Ewa Terczyńska-Dyla, Stephanie Bibert, Francois H. T. Duong, Ilona Krol, Sanne Jørgensen, Emilie Collinet, Zoltán Kutalik, Vincent Aubert, Andreas Cerny, Laurent Kaiser, Raffaele Malinverni, Alessandra Mangia, Darius Moradpour, Beat Müllhaupt, Francesco Negro, Rosanna Santoro, David Semela, Nasser Semmo, Swiss Hepatitis C Cohort Study Group, Markus H. Heim, Pierre-Yves Bochud \& Rune Hartmann

Nature Communications 5:5699 doi: 10.1038/ncomms6699 (2014); Published 23 Dec 2014; Updated 29 Jun 2015

In this Article, Rune Hartman was omitted as joint supervisor of this work. The correct list of joint supervisors is: Markus H. Heim, Pierre-Yves Bochud and Rune Hartmann. 